\title{
Protein-Protein Interaction Analysis through Network Topology (Oral Cancer)
}

\author{
Fazal Wahab Khattak, ${ }^{1}$ Yousef Salamah Alhwaiti, ${ }^{2}$ Amjad Ali, ${ }^{1}$ Mohammad Faisal $\mathbb{D}^{3}{ }^{3}$ \\ and Muhammad Hameed Siddiqi ${ }^{3}$ \\ ${ }^{1}$ Department of Computer and Software Technology, University of Swat, Mingora, KPK, Pakistan \\ ${ }^{2}$ Department of Computer Science, Jouf University, Sakaka, Aljouf, Saudi Arabia \\ ${ }^{3}$ Department of CS \& IT, University of Malakand, Chakdara, KPK, Pakistan
}

Correspondence should be addressed to Mohammad Faisal; mfaisal_1981@yahoo.com

Received 4 November 2020; Revised 21 December 2020; Accepted 23 December 2020; Published 16 January 2021

Academic Editor: Shaukat Ali

Copyright ( 2021 Fazal Wahab Khattak et al. This is an open access article distributed under the Creative Commons Attribution License, which permits unrestricted use, distribution, and reproduction in any medium, provided the original work is properly cited.

\begin{abstract}
Oral cancer is a complex disorder. Its creation and spreading are due to the interaction of several proteins and genes in different biological thoroughfares. To study biological pathways, many high-yield methods have been used. Efforts to merge several data found at separate levels related to biological thoroughfares and interlinkage networks remain elusive. In our research work, we have proposed a technique known as protein-protein interaction network for analysis and exploring the genes involved in oral cancer disorders. The previous studies have not fully analyzed the proteins or genes involved in oral cancer. Our proposed technique is fully interactive and analyzes the data of oral cancer disorder more accurately and efficiently. The methods used here enabled us to observe the wide network consists of one mighty network comprising of 208 nodes 1572 edges which connect these nodes and various detached small networks. In our study, TP53 is a gene that occupied an important position in the network. TP53 has a 113-degree value and 0.03881821 BC value, indicating that TP53 is centrally localized in the network and is a significant bottleneck protein in the oral cancer proteinprotein interaction network. These findings suggested that the pathogenesis of oral cancer variation was organized by means of an integrated PPI network, which is centered on TP53. Furthermore, our identification shows that TP53 is the key role-playing protein in the oral cancer network, and its significance in the cellular networks in the body is determined as well. As TP53 (tumor protein 53) is a vital player in the cell division process, the cells may not grow or divide disorderly; it fulfills the function of at least one of the gene groups in oral cancer. However, the latter progression in the area is any measure; the intention of developing these networks is to transfigure sketch of core disease development, prognosis, and treatment.
\end{abstract}

\section{Introduction}

Verbal cancer, mouth cancer, and head and neck cancer are all related to each other [1], which are cancers affecting cell development or found in the oral cavity [2]. They may occur as an essential injury beginning in any of the tissues within the oral cavity; its reason may be metastasis process occurring away from oral cavity or is due to change in neighbor physical structures, such as the nasal cavity. Or the oral cancers may originate in any of the mouth tissue and can spread to any cell or tissue: teratoma, adenocarcinoma inferred from a saliva gland, and tonsillar or other lymphoid tissues cause lymphoma; pigment-producing cells of the oral mucosa are producer of melanoma. Oral cancers are of few kinds, but almost $90 \%$ originate within the tissues that join the mouth and lips called squamous cell carcinomas [3].

Oral cancer is the most common cancer in the world with low overall survival rates [4], where oral squamous cell carcinoma (OSCC) is the most common type [5]. The organs that suffer from oral cancer include the buccal mucosa, tongue, and lower lip, and it mostly occurs in the people whose age is more than fifty years [6]. Traditional manual 
methods for creating network books, diagrams, or maps have been in use for a long time. The diagrams and other visual data in these valuables were handmade decades ago and have only restricted use. These graphs were based on information of that specific time when created, consisting of unchangeable and interaction less data.

Various biological procedures are enunciated as systems. Networks are demonstrated from the field of atomic sciences such as metabolic networks, protein interaction networks, and gene regulatory networks. Network analysis, sculpting, and visualization are imperative stride about a framework's natural perception of living beings. The visual interpretation of such networks gives the basic idea about the structure of network and also can create an idea about newly generated complex biological data [7].

Genetically, oral cancer has still not been fully analyzed and explored even after several years of research. Many casual or susceptible genes associated with oral cancer have been reported in the research studies. Thus, the focus of our research work is to obtain results that give us useful data related to genes involved in oral cancer and enable us to draw conclusions about gene-gene and related proteinprotein interactions.

\section{Literature Review}

The role of proteins networks in syndromes identification and analyses is very significant. Throughout a period, study in miniature creatures and protein networks plays a vital role in molecular development study enhancement to increase vision in the strength of cells to disruption and also for allotment of new protein occupations. Succeeding those investigations and the present increase of protein interactivity capabilities in mammals, protein networks are progressively helping as toolkit to unknot the molecular foundation of disease. The authors of this paper review favorable uses of protein networks in disease in four main parts:

(a) Latest disease genes identification

(b) Study of their network valuables

(c) Spotting disease-linked subnetworks

(d) Network-based disease sorting

Proteins networks can also be used in areas like infectious disease, self-medication, and pharmacology [8].

Due to interaction detection methods, millions of interactions between proteins have been discovered. Focus of the paper is based on the graph theory, and the authors derived a new method from graph theory known as spectral method. Spectral method was used to disclose unseen networked structures of complex protein-protein collaboration networks. They come up with the idea that these concealed topological layouts comprise organically germane serviceable clusters. This idea stirs a novel approach to forecast the function of unknown proteins based on the classification of known proteins within network structures. With the use of this procedure, 48 quasi-cliques and six quasi-bipartite were separated from a network consisting of
11855 interactions among 2617 proteins in budding yeast, and 76 uncharacterized proteins were allocated functions [9].

All biological processes were considered important and specifically achieved through protein-protein interactions. They use spectral analysis method as a technique to disclose high-level structures using colossal and multifaceted associations. the structurae of the image radius that the authours obtained by the speculative analysis is used in the nalysis for the topology making is used in the analysis for the topology making for the disclosure of unseen topological structures of a complicated interface network [9].

Proteome functional organization can understand better by using human PPI for the identification of interrelating sets of human proteins scientifically; a protein matrix of 4456 baits and 5632 victims was separated by predetermined yeast two-hybrid ( $\mathrm{Y} 2 \mathrm{H})$ interaction coupling. They acknowledged 3186 frequently original relations among 1705 proteins, which produce a huge, highly connected network, following $\mathrm{Y} 2 \mathrm{H}$ technique. The $\mathrm{Y} 2 \mathrm{H}$ scheme is an influential PPIs identification tool, applicable to high-throughput method in detection of complete proteome of organism interactions. To describe high-level confidence relation among proteins, different techniques were used, for example, topological, GO criteria and scoring system. The network was also used to locate genes without any character assigned to humans' disease proteins players in regulating the cellar trials. Screening human proteins relations systematically can enhance the understanding of protein functionality and cellular procedures [10]. They present their experiments results in the form of different graphs and charts.

There are a huge number of collaboration networks; studying them, finding functional nodes and links, and investigating the inner function of cells are the aims of this research. The authors performed a precise chart theorybased examination of this PPI arranged to build computational models for relating and anticipating the properties of deadly changes and proteins contributing in hereditary contacts, utilitarian bunches, protein complexes, and signaling pathways. Their examination suggests that deadly modifications are not as it were exceedingly related inside the organize structure, but they moreover fulfill an extra property. Their evacuation causes a diversion in organized structure. Creators moreover give proof for the nearness of diverse ways that maintain a strategic distance from practical proteins in PPI systems, whereas such ways do not exist for lethal change [11].

The PPI network includes a minor quantity of exceedingly associated protein hubs (also called centers) and various ineffectively associated hubs. Genome-wide consideration shows that cancellation of a center protein is more likely to be hazardous than cancellation of a nonhub protein; a wonder known as the centrality-lethality runs the show. This run of the show is commonly accumulated to reflect the unprecedented centrality of centers in organizing the courses of action of qualities, which in turn suggests the normal importance of organize plans, a key conviction of frameworks of science. Concurring to creators, for the notoriety of this clarification, the fundamental cause of the 
centrality-lethality running the show has never been basically inspected. They propose the concept of basic PPIs to discover out PPIs that are vital for the presence or propagation of a life form. As anticipated, basic PPIs are developmentally more moderated than insignificant PPIs. Considering the part of basic PPIs in deciding quality essentiality, they discover the yeast PPI organize practically more energetic than arbitrary systems, however with less distant sound than the potential ideal. These and other discoveries give unused viewpoints on the organic centrality of arranged structure and vigor [12].

Genomic consideration shows that erasing an exceedingly associated protein hub (center) is more likely to be deadly to a living being than erasing a humble associated hub (nonhub), a strategy recognized as the centrality-lethality running the show. As center points are more imperative than nonhubs in organizing the worldwide arranged structure, the centrality-lethality running the show is broadly accepted to reflect the worth of organized engineering in characterizing organized work, a key idea of frameworks science. Consequently, the centrality-lethality running the show is clarified without the association of organized engineering. Utilizing yeast information, the creators gave down to earth confirmation supporting their speculation [12].

In paper [13], ARIYA et al. suggested a microRNA consisting of multiple genes; actually microRNAs are analyzed, which seemed to be fruitful in high level of tumor. They veiled for the genetic factor; the appearance of these genetic factor remained associated with oral cancer development and evolution. Therefore, recognizing medications that might aim at these genetic factors might benefit plummeting the oral cancer humanity by cultivating development of patients.

In paper [14] by amiri et al., the scheme method has been planned to reconnoiter hereditary difficulty of oral cancer besides recognizing innovative oral cancer associated genes to perceive genomic modifications at molecular smooth, as concluded from variance investigation. Their genetic and communicating examination indicated important enhancement metabolism, motioning trail, and microRNA trails on the road to development. Their integrative method would benefit discovering the genetic variants of oral cancer that can speed up drug detection consequences to mature a healthier sympathetic concerning action approaches for numerous cancer categories.

Protein-protein interaction (PPI) network analysis of cancer has gained focus of medical and biological scientists. Through this approach, examination of the interaction between genes that cause cancer could lead us to improve the diagnosis and treatment of patients $[15,16]$. In PPI network analysis, the genes related to the disease are gathered and organized in an integrative structure $[15,17]$. The calculation of topological properties of the network, including central parameters such as degree and centrality, provides useful information about molecular mechanism of disease onset and pathology [18]. Introducing selected genes among large number of query genes can lead to specific biomarker panel related to the disease [19].

\section{Methodology}

The methodology of protein-protein interaction of oral cancer consists of seven steps. The first step is the attainment of the candidate genes of oral cancer, which was done in two ways: Firstly, PolySearch text-mining system is used, which is a web-based tool exploiting various techniques to highlight and align informative text. PolySearch results gave us a large list of genes and proteins. A lot of data are available on cancer. As our specific target is to collect and analyze data on oral cancer, PolySearch tool is used for obtaining the specific data on oral cancer. Secondly, we manually confirmed from reviewing the literature that the obtained genes and proteins were indeed of oral cancer. The second step of the methodology involved the use of STRING database for scanning the protein interactions. Construction of PPI networks, from which an extended network was derived, was used to extract the giant component making up the third step. The fourth step involved the analysis of the PPI network based on its topology. The fifth step was the creation of a backbone network from the giant network based on the highest betweenness centrality (BC) value. The sixth step involved the construction of a subnetwork from the giant network that consisted of all shortest routes among the aspirant genes. The seventh step concluded with TSPO as central protein.

\subsection{Dataset Preparation for the Extraction of Genes Associated} with Oral Cancer. Candidate genes associated with oral cancer were searched using PolySearch text-mining system, which is a useful tool in producing a list of ideas based on the query of the user. PudMed, OMIM, Drug Bank, and SwissProt are among the few sources of information from which data relevant to the query can be extracted. PolySearch contributes a great deal to extracting information on important biomedical concepts such as genes/proteins, disease, SNPs, drugs, metabolites, pathways, and tissues, which could help in finding the relevant genes of oral cancer [20]. This method is used for finding genes related to oral cancer. Search was performed using the key words "Disease with Gene/Protein Association" and "Oral Cancer." The literature analysis by PolySearch system returned 274 results. To determine the accuracy, these results were confirmed manually to check whether oral cancer associated genes were viable candidates or not. Discarding the less significant genes resulted in a total of 208 candidate genes for oral cancer by literature survey (Table 1).

3.2. Scanning Protein-Protein Interactions. Oral cancer candidate genes listed in Table 1 were converted to seed proteins. For visualizing the PPIs, STRING database was used. It is an exploratory database for PPIs. Updated version of STRING, 9.1, contains more than 1100 organisms, covering millions of proteins [20].

3.3. Constructing PPI Network from the Extension for Extraction of the Giant Component. A system, which consisted of the seed proteins and has also direct association with their 


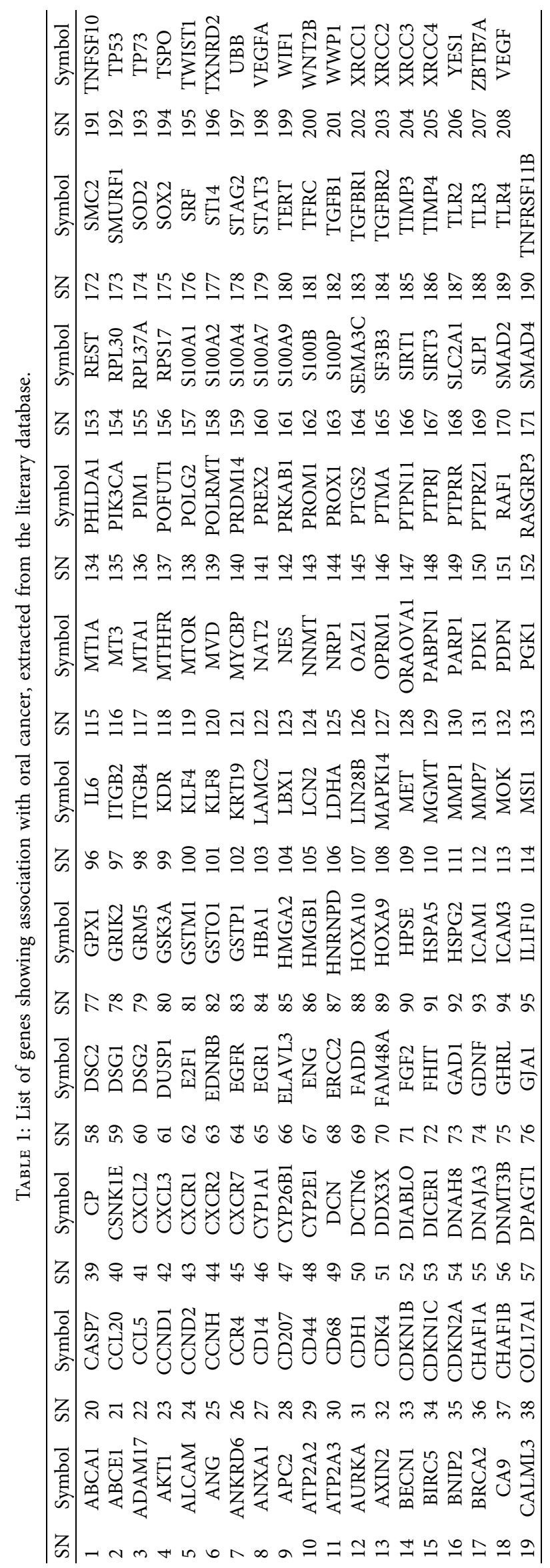


PPI's neighbors, was developed. This system was built using Cytoscape [21], which is an extremely flexible project designed for the purpose of investigations, operations, and visualization of extensive systems. Not only did this study involve expansion on the system incorporating a giant part but also it included two small separate parts. The expectation of this study was that the giant network must consist of hubs with extensive $\mathrm{BC}$ value. This was clearly in light of the fact that both separate parts consist of a small number of hubs, and for this purpose the investigation and preparation involved only the giant network with its various parameters. The use of giant system obtained from the extended system was examined and analyzed by this method advantageously.

3.4. Protein Interaction Network Involving Topological Analysis. For accessing the hubs in the system, nodes properties such as betweenness centrality (BC), closeness centrality (CC), and degree (K) were noted and used, especially K and BC values. Quantity of adjacent joints which is based on the determination of the number of connections of one protein to its neighbors determines the most important factor, that is, the degree (K). The number of shortest routes that pass through each node, thus measuring the frequent occurrence of the node which has the greatest restricted routes between other nodes, determines the betweenness centrality. Shortest path or the most limited way is found by measuring the length of every last one of the geodesics to or from the network. The flow in the network is characterized by a high $\mathrm{BC}$ node value to great extent. $\mathrm{BC}$ may assume an integral part as a global property, since it is a valuable pointer to distinguish bottlenecks in a system. The converse of the normal length of the briefest paths among all the other nodes in the graph, which let us know the topological focus of the network, constitutes a closeness centrality (CC). Estimates of networks based on global topology include average degree, mean most brief path length, and distances utilized in the network.

Average degree $(<\mathrm{K}>)$ : mean of total degree values of nodes in a network

Mean shortest path length (mspl): represents connecting average of the steps involved to every pair of nodes using their shortest path

Diameter (D): longest paths among all shortest paths, characteristics of nodes, and measurements used to characterize the network were calculated by Cytoscape software in this paper

3.5. Creating Backbone Network by Searching High BC. This study involved observing the PPI supporting the oral cancer-related gene network.

Consequently, the proteins with high $\mathrm{BC}$ ought to have vigorously utilized crossing points as the backbone actually consisted of these proteins and their connections within the network. The critical point with high $\mathrm{BC}$ value was set at top 15 genes of the total number of nodes within the network $[9,10]$. BC nodes with high values and their interlinkage were mined utilizing the giant network for creation of a backbone network. Furthermore, BC also served the purpose of measuring the nodes centrality in the network initially since according to the definition, $\mathrm{BC}$ values that are high consist of the shortest paths in the network passing through the nodes. These nodes with high BC value are among other nodes of the network function as bottleneck control interaction.

3.6. Construction of a Subnetwork among the Candidate Genes with the Shortest Paths. Some of the candidate genes in the network are not directly connected and that includes the giant network as well. For constructing a subnetwork, genes connected with least number of nodes, in link with oral cancer, are required. For this, each combination of candidate qualities and their most limited way was calculated. These ways were found using Cytoscape software, coming about within the subnetwork comprising all nodes in these ways.

3.7. Central Protein TP53 Validates the Backbone Network. Validation was essential for checking the healthiness of the backbone network. For this, test networks were developed as they were employing a little component of 208 genes as initial seeds. For this, once more Cytoscape computer program was utilized. BC value was determined in these test networks. Segments having nodes with top 15 $\mathrm{BC}$ in the test networks agreed with the nodes in the backbone network as per description in the fifth step to determine the accuracy of the backbone. Furthermore, the healthiness of backbone network of oral cancer proteins was determined by calculation of the frequency of the nodes having the largest $\mathrm{BC}$ value which gives the accuracy of the backbone nodes.

\section{Results}

In this research work, we have obtained protein-protein interaction network through applying various steps discussed in the Methodology section. Then, we have obtained the key nodes involved in protein-protein interaction network and perform the analysis for obtaining important protein or genes involved in oral cancer.

4.1. Network with Protein-Protein Interactions. A huge network with several collapsed smaller networks, respectively, found from the seed proteins TSPO and TP53, was also a part of the extended network (Figure 1). Giant network contained of 208 nodes connected with 1572 edges as illustrated in (Figure 2). The backbone network, however, comprised 322 edges linking 29 nodes (Figure 3). Another network consists of 25 nodes linking 87 edges (Figures 4 and 5). The further distinguishing feature is the presence of some very highly connected nodes, though they are very small in number, while others show fewer connections. 


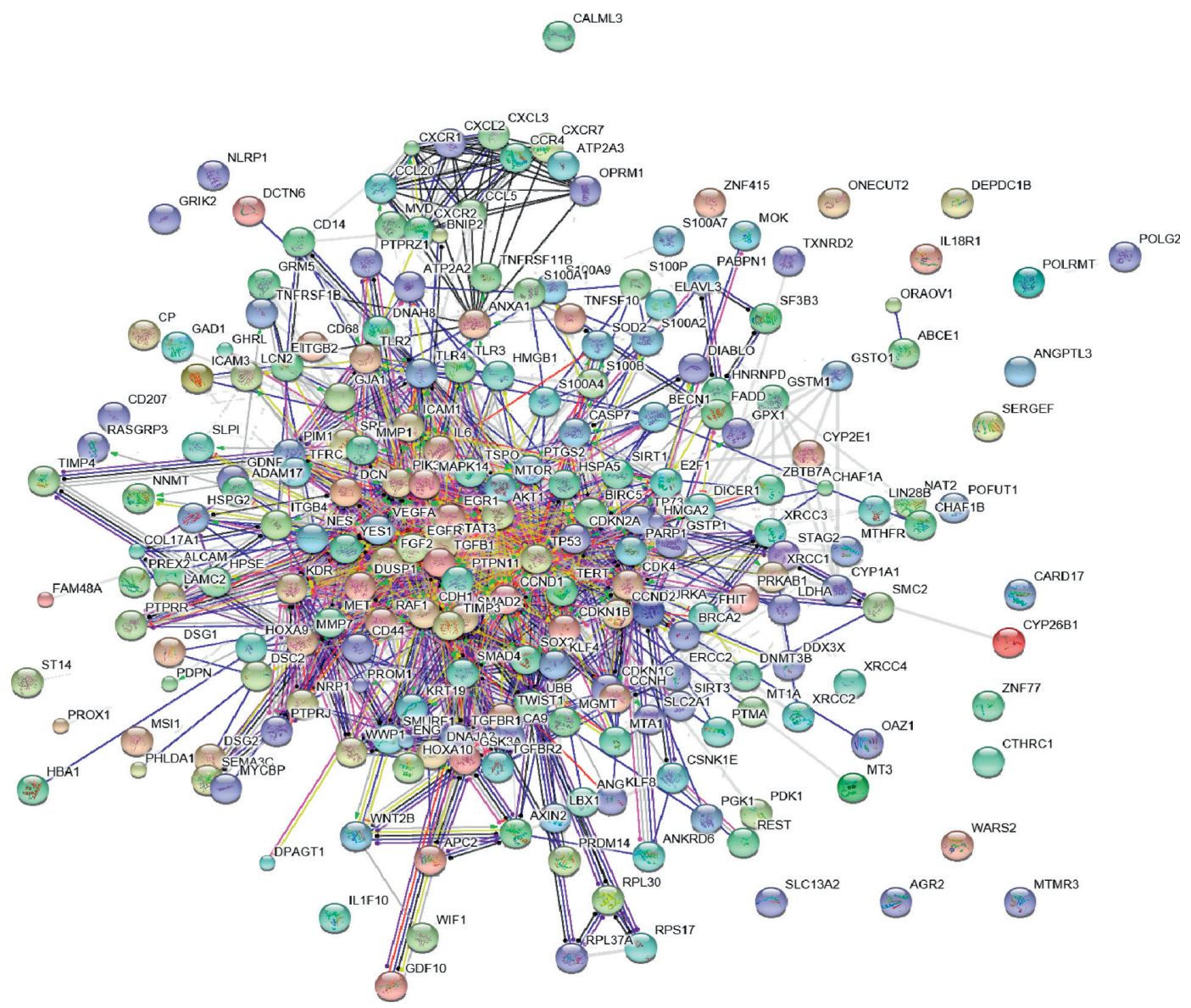

FIGURE 1: Illustration of the extended network, which includes the huge network and some smaller networks. The labeled nodes are known as seed proteins, which were within the list of candidate genes, shown in Table 1.

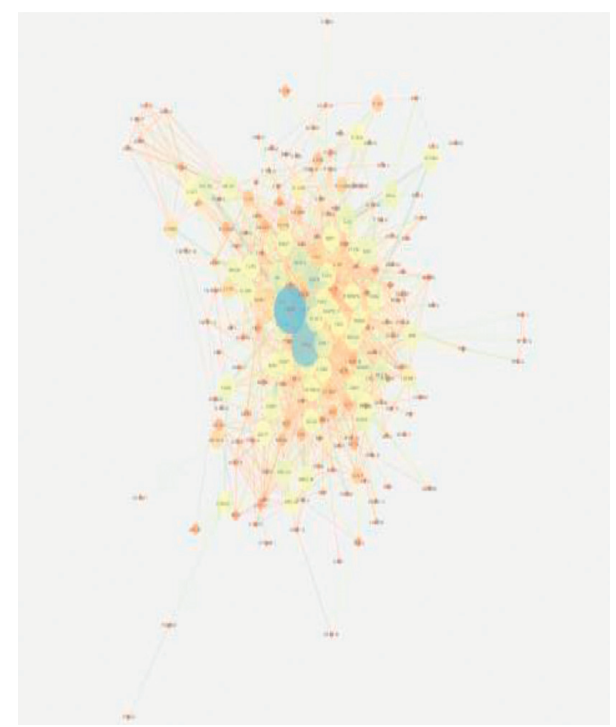

Figure 2: Illustration of giant network topology. This huge network, which was derived from the extended network, is its biggest segment. $\mathrm{BC}$ values indicate the size of nodes.

4.2. Analysis of the Key Nodes Involved in the PPI Network. Key nodes were viewed by using nodes having large degree or high betweenness centrality values, and the critical point was set as $10 \%$ of the total number of nodes for both the degree and BC in the network in this study. Among the total 208 nodes, those that had high BC were 59 in number, and 


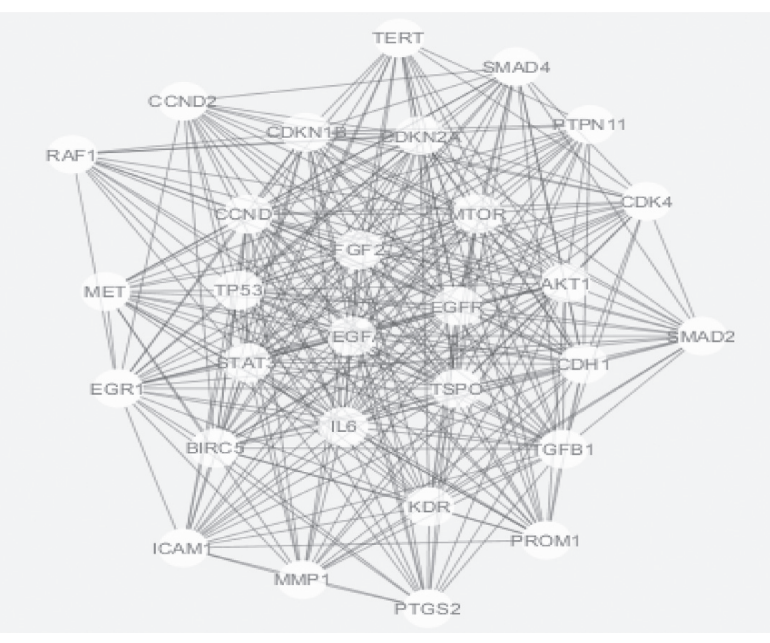

FIgURE 3: Illustration of the backbone obtained from other networks. It consists of 29 nodes with highest betweenness centrality, and nodes' size indicates their $\mathrm{BC}$ values.

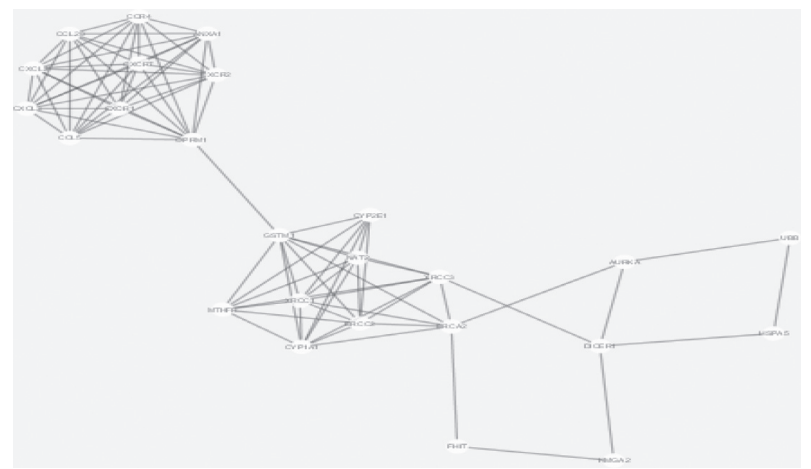

Figure 4: Illustration of the backbone obtained from other networks. It consists of 25 nodes and 87 edges.

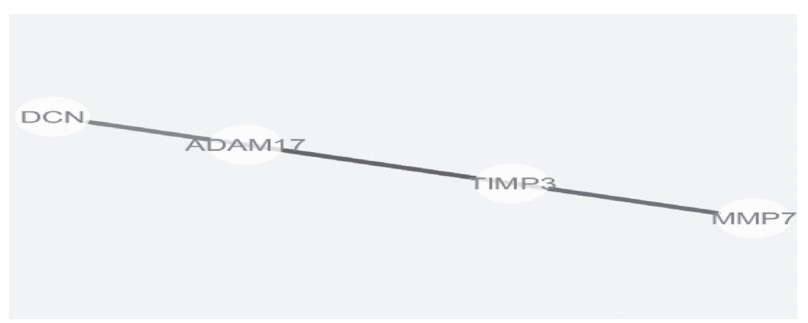

FIGURE 5: Illustration of the backbone obtained from other networks. It consists of 4 nodes and 5 edges with highest betweenness centrality.

109 nodes had a large degree. 10 nodes had a large degree and high betweenness centrality (Table 2), nodes among the high $\mathrm{BC}, \mathrm{CC}$, and degree nodes (Table 3). To visualize the role of each protein in the oral cancer network, different colors and sizes were assigned to highlight them (Figure 2). TP53 is a protein with largest degree, TPSO is a protein with highest $\mathrm{BC}$ value, and EGFR is a protein with highest CC value. TP53 occupies central position in the network due to its high degree, $\mathrm{BC}$, and CC value. Signaling pathways in the high betweenness centrality network and their cross-talks
TABLE 2: List of the proteins having high $\mathrm{BC}$ and large degree values.

\begin{tabular}{lccc}
\hline SN & Gene & Degree & BC \\
\hline 1 & TSPO & 93 & 0.04809981 \\
2 & TP53 & 113 & 0.03881821 \\
3 & AKT1 & 90 & 0.01744922 \\
4 & EGFR & 79 & 0.01611723 \\
5 & VEGFA & 60 & 0.01249361 \\
6 & IL6 & 60 & 0.00829916 \\
7 & MTOR & 49 & 0.00784171 \\
8 & CDH1 & 66 & 0.00782314 \\
9 & CXCR2 & 17 & 0.00712798 \\
10 & SMAD4 & 39 & 0.00692481 \\
\hline
\end{tabular}

TABle 3: Nodes with high BC, CC, and degree values.

\begin{tabular}{lccccc}
\hline Gene & Degree & Gene & CC & Gene & BC \\
\hline TP53 & 113 & EGFR & 0.75409836 & TSPO & 0.04809981 \\
TSPO & 93 & MAPK14 & 0.75 & TP53 & 0.03881821 \\
AKT1 & 90 & CDKN1B & 0.75 & AKT1 & 0.01744922 \\
EGFR & 79 & CDK4 & 0.73684211 & EGFR & 0.01611723 \\
CDH1 & 66 & TSPO & 0.69767442 & VEGFA & 0.01249361 \\
CCND1 & 61 & STAT3 & 0.6875 & IL6 & 0.00829916 \\
VEGFA & 60 & IL6 & 0.67741935 & MTOR & 0.00784171 \\
IL6 & 60 & CD68 & 0.66666667 & CDH1 & 0.00782314 \\
STAT3 & 56 & CHAF1A & 0.66666667 & CXCR2 & 0.00712798 \\
FGF2 & 53 & OAZ1 & 0.66666667 & SMAD4 & 0.00692481 \\
MTOR & 49 & EGR1 & 0.64705882 & XRCC3 & 0.00531283 \\
MAPK14 & 49 & FGF2 & 0.63829787 & MET & 0.00516178 \\
CDKN2A & 47 & BIRC5 & 0.63513514 & UBB & 0.00501709 \\
TGFB1 & 47 & MMP1 & 0.63218391 & PARP1 & 0.00451757 \\
KDR & 42 & RAF1 & 0.63157895 & GJA1 & 0.00447296 \\
\hline
\end{tabular}

are obtained from the backbone networks. 1st backbone network consists of 29 nodes and 322 edges (Figure 3). Second major backbone network consists of 25 nodes and 87 edges (Figures 4 and 5). Analyzing the values of $\mathrm{BC}$ and CC shows that TP53 locates at the center of the backbone network with the highest $\mathrm{BC}$ value and the largest degree. TP53 has 29 neighbors: CCND1, SMAD2, TSPO, MTOR, ICAM1, VEGFA, STAT3, EGR1, TERT, MMP1, CCND2, MET, AKT1, KDR, TP53, RAF1, IL6, EGFR, PTGS2, PTPN11, FGF2, CDKN2A, TGFB1, BIRC5, CDKN1B, PROM1, SMAD4, CDH1, and CDK4. Details of other proteins in the backbone network were not included here.

\section{Discussion}

Genetically oral cancer has not been fully explored; even after several years of research it still remains unexplored. Many casual or susceptible genes associated with oral cancer have been reported. The multiple factors contributing to hepatocarcinogenesis include ecological, transmissible, sustaining, metabolic, and endocrine structures, which are involved specifically or indirectly in the development of oral cancer. The oral cancer pathogenesis indicates the involvement of several genes and can broadly be classified into four majors groups: regulatory genes involved in response to DNA damage, cell cycle control players, those intricate 
growth inhibition, apoptosis, and also the genesis involved in cell-cell collaboration and signal transduction. Analyzing the contribution of genes and proteins pertaining to oral cancer pathogenesis in addition to the involvement of other key proteins in the PPI networks by analyzing their topology was the goal of this study. The results obtained gave us useful data related to genes involved in oral cancer and enabled us to draw conclusions about gene-gene and related proteinprotein interactions. It was observed that most of the seed proteins (208) connected to giant network in terms of oral cancer and their PPI neighbors. This giant oral cancer network consisted of 1572 edges. Backbone network topology, however, resulted in different small network topologies. As it is not possible to draw conclusion from the giant network, we further split down the backbone network into small subnetworks for obtaining clear results on oral cancer. The splitting of backbone network is performed according to properties of nodes, that is, BC, CC, and degree values. One backbone network consists of 29 nodes and 322 edges. Another subnetwork consists of 27 nodes 87 edges. There is one small subnetwork of 4 nodes and 5 edges. Other subnetworks also exist but they are very small. Literature was analyzed to find further techniques and methods supporting the importance of different genes in oral cancer. TP53 is a gene that occupied important position in the network. TP53 has degree value of 113 and $B C$ value of 0.03881821 . Literature was analyzed to find further techniques and methods supporting the importance of TP53 in cancer. In this regard, researchers have clarified TP53 changes in different types of cancers. Moreover, it has been discovered that TP53 reaction pathway is regularly flawed in human diseases and the recurrence of TP53 fluctuates greatly. In another study conducted by Ding and colleagues, polymorphisms were affiliated with this disorder. The study was focused around metainvestigation to better comprehend the relationship between polymorphism of Condon 72 and the tumor protein p53 (TP53) gene, which results in a missense transformation of arginine (R) to proline $(\mathrm{p})$ and causes vulnerability to hepatocellular carcinoma $[22,23]$. TSPO is another important gene in oral cancer network having highest BC value of 0.04809981 and degree value of 93 . TP53 mutation sites with stalk cell-like gene appearance in addition to association with many cancers were also found in oral cancer with high mortality rate, although the exact sites are unknown as of yet. Moreover, direct sequencing methods are more helpful for reliable results. Form direct sequencing result, it was concluded that TP53 mutations, mainly the blistering mutations R249S and V157F, are linked with meager prognosis for patients with oral cancer. The stem cell-like acquirement of gene manifestation traits might be a contributing factor to the violent behavior of tumors with TP53 mutations having shorter survival rate as compared to wild-type TP53, according to literature survey [23-25].

\section{Conclusion}

The mainstay network shows clearly all the essentials genes of oral cancer, their associated regulatory pathways, and the interactions between them. This eventually brought us to the conclusion that TP53 is the protein with the highest degree, TSPO is the protein with largest $\mathrm{BC}$, and EGFR is the protein with highest CC values, but TP53 obtained the important position in the network due to its degree, BC, and CC values, indicating that TP53 is centrally localized in the network and is a significant bottleneck protein in oral cancer protein-protein interaction network. These findings suggested that pathogenesis of oral cancer variation was organized by means of an integrated PPI network, which is centered on TP53. Furthermore, our identification of TP53 as the bottleneck protein in oral cancer network determined its significance in cellular network in the body as well, as P53.

Tumor protein 53 is important in the regulation of cell division; by restricting the cells from growing or dividing uncontrollably, it fulfills the function of at least one of the gene groups in oral cancer.

\section{Data Availability}

The data used to support the findings of this study are included within the article.

\section{Conflicts of Interest}

The authors declare that they have no conflicts of interest.

\section{References}

[1] R. Lozano, M. Naghavi, K. Foreman et al., "Global and regional mortality from 235 causes of death for 20 age groups in 1990 and 2010: a systematic analysis for the global burden of disease study 2010," The Lancet, vol. 380, no. 9859, pp. 2095-2128, 2013.

[2] J. W. Werning, Ed., Oral Cancer: Diagnosis, Management, and Rehabilitation, Thieme, New York, NY, USA, 2011.

[3] A. C. Chi, T. A. Day, and B. W. Neville, "Oral cavity and oropharyngeal squamous cell carcinoma-an update," $C A A$ Cancer Journal for Clinicians, vol. 65, no. 5, 2015.

[4] B. Wang, S. Zhang, K. Yue, and X.-D. Wang, "The recurrence and survival of oral squamous cell carcinoma: a report of 275 cases," Chinese Journal of Cancer, vol. 32, no. 11, pp. 614-618, 2013.

[5] C. Rivera, "Essentials of oral cancer," International Journal of Clinical and Experimental Pathology, vol. 8, no. 9, pp. 11884-11894, 2015.

[6] S. D'souza and V. Addepalli, "Preventive measures in oral cancer: an overview," Biomedicine and Pharmacotherapy, vol. 107, no. 3, pp. 72-80, 2018.

[7] A. Kerren and F. Schreiber, "Network visualization for integrative bioinformatics," in Approaches in Integrative Bioinformatics, pp. 173-202, Springer, Berlin, Germany, 2014.

[8] T. Ideker and R. Sharan, "Protein networks in disease," Genome Research, vol. 18, no. 4, pp. 644-652, 2008.

[9] D. Bu, Y. Zhao, L. Cai et al., "Topological structure analysis of the protein-protein interaction network in budding yeast," Nucleic Acids Research, vol. 31, no. 9, pp. 2443-2450, 2003.

[10] U. Stelzl, U. Worm, M. Lalowski et al., "A human proteinprotein interaction network: a resource for annotating the proteome," Cell, vol. 122, no. 6, pp. 957-968, 2005. 
[11] N. Pržulj, D. A. Wigle, and I. Jurisica, "Functional topology in a network of protein interactions," Bioinformatics, vol. 20, no. 3, pp. 340-348, 2004.

[12] X. He and J. Zhang, "Why do hubs tend to be essential in protein networks?” PLoS Genetics, vol. 2, no. 6, p. e88, 2006.

[13] S. Ariya, A. James, and B. Joseph, "Computational analysis of oral cancer gene expression profile and identification of MiRNAs and their regulatory hub genes," Journal of Complementary Medicine Research, vol. 11, no. 3, pp. 154-159, 2020.

[14] N. Amiri-Dashatan, M. Koushki, A. Jalilian, N. A. Ahmadi, and M. Rezaei Tavirani, "Integrated bioinformatics analysis of mRNAs and miRNAs identified potential biomarkers of oral squamous cell carcinoma," Asian Pacific Journal of Cancer Prevention, vol. 21, no. 6, pp. 1841-1848, 2020.

[15] H. Zali and M. Rezaei Tavirani, "Meningioma protein-protein interaction network," Archives of Iranian Medicine, vol. 17, no. 4, pp. 262-272, 2014.

[16] N. Safari-Alighiarloo, M. Rezaei-Tavirani, M. Taghizadeh, S. M. Tabatabaei, and S. Namaki, "Network-based analysis of differentially expressed genes in cerebrospinal fluid (CSF) and blood reveals new candidate genes for multiple sclerosis," PeerJ, vol. 4, Article ID e2775, 2016.

[17] H.-A. Abbaszadeh, A. A. Peyvandi, Y. Sadeghi et al., "Er: YAG laser and cyclosporin a effect on cell cycle regulation of human gingival fibroblast cells," Journal of Lasers in Medical Sciences, vol. 8, no. 3, pp. 143-149, 2017.

[18] M. Rezaei-Tavirani, M. Rezaei-Tavirani, V. Mansouri et al., "Introducing crucial protein panel of gastric adenocarcinoma disease," Gastroenterology and Hepatology from Bed to Bench, vol. 10, no. 1, pp. 21-28, 2017.

[19] M. Rezaei Tavirani, F. OkHOVATIAN, M. Zamanian Azodi, and M. Rezaei Tavirani, "Duchenne muscular dystrophy (DMD) protein-protein interaction mapping," Iranian Journal of Child Neurology, vol. 11, no. 4, pp. 7-14, 2017.

[20] J. Li, M. Nazir Jan, and M. Faisal, "Big data, scientific programming, and its role in internet of industrial things: a decision support system," Scientific Programming, vol. 2020, Article ID 8850096, 2020.

[21] X. Liao, M. Faisal, Q. QingChang, and A. Ali, "Evaluating the role of big data in IIOT-industrial internet of things for executing ranks using the analytic network process approach," Scientific Programming, vol. 2020, Article ID 8859454, 2020.

[22] D. Cheng, C. Knox, N. Young, P. Stothard, S. Damaraju, and D. S. Wishart, "PolySearch: a web-based text mining system for extracting relationships between human diseases, genes, mutations, drugs and metabolites," Nucleic Acids Research, vol. 36, pp. W399-W405, 2008.

[23] P. Shannon, A. Markiel, O. Ozier et al., "Cytoscape: a software environment for integrated models of biomolecular interaction networks," Genome Research, vol. 13, no. 11, pp. 2498-2504, 2003.

[24] C. Ding, H. Yu, H. Yu, and H. Qin, "TP53 codon 72 polymorphism with hepatocellular carcinoma: a meta-analysis," Journal of International Medical Research, vol. 40, no. 2, pp. 446-454, 2012.

[25] H. G. Woo, X. W. Wang, A. Budhu et al., "Association of TP53 mutations with stem cell-like gene expression and survival of patients with hepatocellular carcinoma," Gastroenterology, vol. 140, no. 3, pp. 1063-1070, 2011. 